\title{
Digital Innovation Hubs for Enhancing the Technology Transfer and Digital Transformation of the European Manufacturing Industry
}

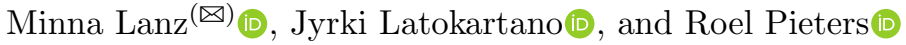 \\ Tampere University, Tampere, Finland \\ minna.lanz@tuni.fi \\ https://www.tuni.fi
}

\begin{abstract}
The European manufacturers are dealing with shorter product life-cycles and smaller batch sizes. Especially, the high-value products tend to be fully personalised, which makes the automatisation of the production difficult. However, the trend is that the production needs to be predictable and fully traceable to the process and even to the tool level. This adds pressure to have better data collection methods and also to increase of automation in different levels of production. The emergence of new technologies in the field of robotics allows the utilisation of automation in flexible manner. Within all areas of robotics, the demand for collaborative systems is rising as well. The level of desired collaboration and increased flexibility will only be reached if the systems are developed as whole e.g. perception, reasoning and physical manipulation. However, at the same time there is concerns on how to attract capable personnel to the factories. In order to fully implement and utilise the new robotics technologies the industry needs capable resources. For answering these needs there has been several attempts to build different types of industrial ecosystems to facilitate better the technology and knowledge transfer, and share of expertise. The main aim of the paper is to review recent actions regarding the robotics projects forming industrial ecosystems in the Horizon 2020 framework programme, and then introduce the TRINITY Digital Innovation Hub (DIH) project approach to form an industrial ecosystem in the field of robotics.
\end{abstract}

Keywords: Digital innovation hubs $\cdot$ Robotics $\cdot$ ICT $\cdot$ Skills

\section{Introduction}

The demand for industrial robots (market) is anticipated to be growing to 65 billion Euros by the year $2023[13,31,34]$. At the same time there is a rising need for more flexibility and collaboration with robots and humans in the factory floor. The need for collaborative robots in the automation industry is acting as 
a driver for this market and is expected to serve as a market opportunity for future growth. In addition, various countries across the world are reviving their electronics and consumer goods industry, which is in turn contributing to the growing demand for collaborative robots. The continued rise of industrial robots certainly seems to be an inevitability being driven by a variety of production demands, including the need for safer and more 'simplified' robotic technologies to work in collaboration with humans, increased resource efficiency, and continued adaptation to the proliferation of automation and the IoT $[13,31]$.

The use of industrial robots in large manufacturing companies is generally well established and understood. However, In 2015, enterprises employing fewer than 250 persons represented $99 \%$ of all enterprises in the EU. In manufacturing the SMEs employ $57 \%$ of workforce [11]. In order to stay competitive, smaller scale and SME manufacturing need to embrace smart robotics to maintain efficiency and create jobs. Raising the output and efficiency of SME manufacturers will have a significant impact on Europe's manufacturing and employment capacity. In turn this will increase overall employment as companies expand into markets considered inaccessible given Europe's comparative labour costs. Increasingly Europe will not only be competing against low wage economies but also, increasingly, highly automated ones. Leadership in secure robotics technology will be a key differentiator of market share in many sectors.

\subsection{Emerging Robotics Trends}

The term 'robot' has also been undergoing a major change in the recent years. Robots are no longer standalone operation units such as in the 80 's. Today a robot is a part of a bigger system with multiple interfaces and related components such as flexible fixtures and grippers. Robots receive commands from the internet and may even have no fixed sensors on their mechanical body, rather a combination of external sensors providing feedback to the mechanical system directly or indirectly. The term 'robot' includes digital aspect, as robots embedded in cars/consumer electronics/mobile phones and any electronic appliances that can communicate through internet or other wired/wireless communication. This offers a huge business potential [20]. The next-generation robots that have the potential to realise agile production processes are based on transformation of industrial robots to something that everyone can accept, understand, and utilize [13].

Human safety raises an issue. The footprint of the safety protected robot cells are large, and static safety measures prevent reconfiguration of the cells, thus reducing the efficiency of work, especially small batch-size production. Noteworthy, the human-robot interaction research has been focusing world-wide on small, "human friendly" robots or inherently safe robot systems, such as Kuka iiwa, ABB YuMi and Baxter [6]. However, the future robotic systems will need carry also large loads with long reach to serve better the European manufacturing industry [15]. These systems are designed to carry large loads with maximal speed and a long range and at the same time the systems need to be highly re-configurable [22] and adaptable to changing customer needs [27]. The level of 
desired collaboration and increased flexibility will only be reached if the systems are developed as a whole including perception, reasoning and physical manipulation. Industrial manufacturing is going through a process of change toward flexible and intelligent manufacturing, the Human-robot collaboration (HRC) will have a more prevalent role in future [26].

Traditionally fences prevent the operators from moving into the robots' working area. This evolution means breaking with the established safety procedures as the separation of workspaces between robot and human operator is removed [18]. Also due to economic reasons, there is a need to decrease the factory footprint, allow existence of shared workplaces, and maintain and reconfigure the production environment while production is running $[15,36]$. Hence, when considering especially the large-sized robots with significant mass of inertia, all sensory solutions that help robots react to danger are useful now, not just years from now.

\subsection{Ecosystems}

Based on the discussion and vision papers from the European technology platforms and communities, the innovation ecosystems will play major role in future research, development and innovation developments. In the visions the ecosystems are expected to contribute to economy and business creation from advanced technologies, building of innovation ecosystems, and facilitating the skills development in Europe [2,25].

Big Data Value Association and euRobotics [2] in their joint vision expect Innovation Ecosystems to have following features:

- European focal point for exchange and coordination of the AI, Data and Robotics innovation communities

- National and regional alignment

- Engagement of Stakeholders

Manufacture Vision 2030 [25] described the future manufacturing ecosystems to be complex, multi-facetted, highly networked and, dynamic socio-technical system. The emerging technologies such as Artificial Intelligence (AI) and Digital Security and Connectivity increase the vertical and horizontal integration, considering the whole life cycle of manufactured products. Physical products and service offerings are fully integrated into the lifecycle, including design and engineering, embedded systems, process support systems, production technology and support services. AI will enable increased levels of automation and human interaction, while Cyber Security will be a prerequisite for global collaboration and interaction. In this broad ecosystem, the manufacturing impact and strategic importance can be measured in terms of value added and created direct and indirect jobs.

\subsection{Future Skills}

In small and medium-sized enterprises (SMEs), training needs arise from the increased use of modern digital manufacturing tools, cybersecurity new additive 
manufacturing processes and novel engineering of intelligence solutions. As a direct result, workers need to develop new skills and competences to work effectively [16]. From an educational perspective. It is especially critical that people with few prior successful experiences in fully applying the key informationprocessing skills should obtain adequate comprehension to guide them in structural changes in their future working lives. Human capital is the main enabler for adopting the emerging technologies such as robotization and digitalization [23]. However, the number of science enrolments and graduates in Europe has been slowing down over the last decade, declining from $24.3 \%$ in 2002 to $22.6 \%$ in 2011 according to Eurostat. The males still predominate the field of engineering, by representing more than $80 \%$ of graduates in particular in the fields of computing and engineering [10].

The key challenge in addressing the evolution of future education in the manufacturing sector involves developing skills and expertise as well as pedagogical and technological approaches that match the changing needs of today's and future workplaces [3,25]. While the discussion is indeed about the skills gap and how to improve existing skills, there is little attention paid to demographics. The population of Europe is not growing, and the decrease is expected to start around 2040-2050 in Europe [14].

\section{Review of Digital Innovation Hubs in Robotics}

The Digital Innovation Hubs are expected to have both a local and European functioning. Member States will be expected to co-invest in the hub through funding the facilities and services with a local impact in their regions/country, whereas the European dimension (opening up the facilities to all of Europe and importing missing expertise) will be funded through a grant of Digital Europe. The main reason for developing the DIH concept is to facilitate the digitalisation of the European industry. The level of digitalisation of industry remains uneven, depending on the sector, country and size of company. Many organisations still struggle to make the most of digital opportunities due to lack of knowledge or difficulties to find finance [9]. The need for enhanced technology transfer is highlighted in both ManuFuture [25] and EuRobotics [2] Vision documents.

During the H2020 Framework Programme several Digital Innovation rampup projects were funded. The Fig. 1 shows the list of funded and/or currently running project. The state of the art review is based on material found from Cordis database [4]. The keywords used to find robotics projects were "robot", "robotics", "fstp", "cascade", "re-configuration", "automation". The search was limited projects in Horizon 2020 programme within the H2020 Industrial Leadership - Leadership in enabling and industrial technologies - Information and Communication Technologies (ICT).

Based on the review there is a number of Digital Innovation Hubs emerging offering funding for SMEs with slightly different manners. The projects maturity level is defined by the call, which is Innovation Action. The expected technology readiness level (TRL) is mainly between 5 and 7 , where the TRL5 stands for 


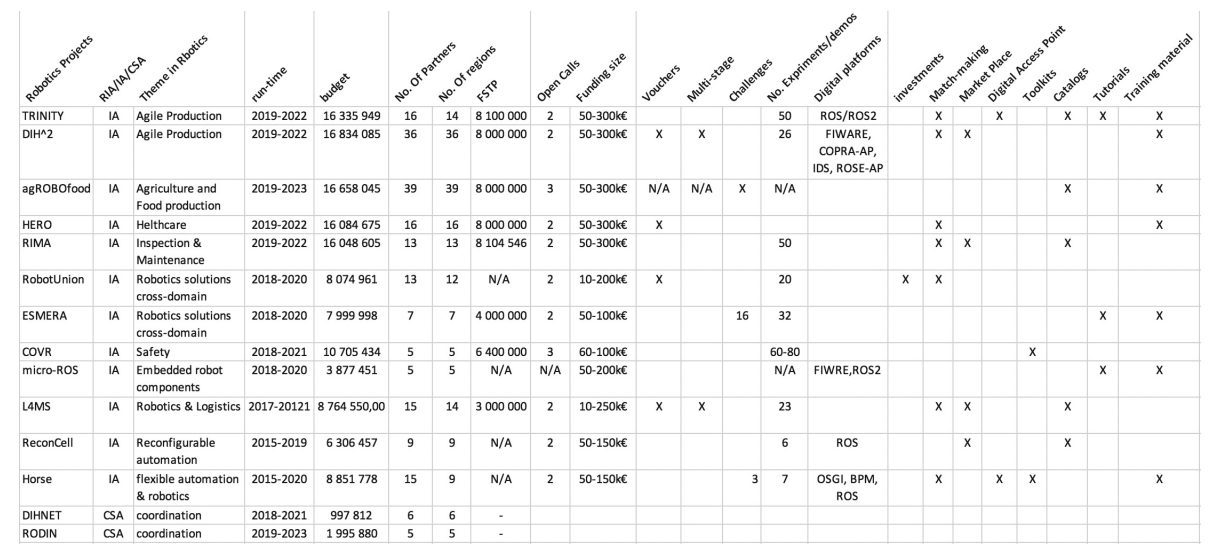

Fig. 1. The analysis of DIH activities within past 5 years

'technology validated in relevant environment (industrially relevant environment in the case of key enabling technologies)' and TRL7 for 'system prototype demonstration in operational environment'. In some cases the demonstrations can reach TRL 8 e.g. demonstration called in RobotUnion [32]. Most of these are dedicated to publish smaller demonstrators that include training and set-up material to be used by companies later on. The Horse [19], L4MS [24] and Reconcell [29] were funded under ICT Innovation for Manufacturing SMEs (I4MS) where the special focus was on robotics applications. ESMERA [12], COVR [5] and RobotUnion [32] had the special focus on System abilities, SME and benchmarking actions, safety certification. The TRINITY [35], DIHsquared [7], agROBOfood [1], RIMA [30] and DIH-Hero [17] were funded under H2020 Industrial Leadership with the focus on building Digital Innovation Hubs for Robotics, with the special request for developing a marketplace and community for disseminating and exploiting results post-project. The Coordination and Support Actions (CSA) RODIN [33] and DIHNET [8] are mentioned as they intent to facilitate the collaboration among the cascade funded projects.

\section{Trinity DIH - Concept and Approach}

The main objective of TRINITY is to create a network of digital innovation hubs (DIHs) composed of Research Centers and University Groups specialised in Advanced Robotics and Internet of Things (IoT), supported by a DIH with experts in Robotics Cyber security to contribute to novel robotics solutions that will increase agility in production. The second objective is to continue this network after the ramp-up phase, by building a sustainable business model throughout the project lifetime. The third objective is to deliver a critical mass of use case demonstrations in collaboration with industry to support the industrial modernisation leading to more agile production and increase the competitiveness of 
European companies. The modular and reconfigurable use case demonstrations will show how to combine robotics, IoT and Cybersecurity together. Furthermore, TRINITY will contribute to answer the European Industry demand for advanced, highly flexible and collaborative robotic solutions to keep companies competitive. TRINITY aims to bring together top researchers and industry from all over Europe with the objective of developing new, digital and human oriented robotic technology for improving agility of European manufacturing and innovation capabilities.

\subsection{Use-Case Demonstrations}

The TRINITY-project aims to deliver 50 use-case demonstrations to the market. Each of the demonstrations include different technical solutions, called modules. 20 of these are internal demonstrations that are openly disseminated. Part of these demonstrations are fully open source. The idea is that each module interfaces are described as public specification document. Each internal use-case demonstration module will in the end include set-up tutorials and training material associated to that particular technology.

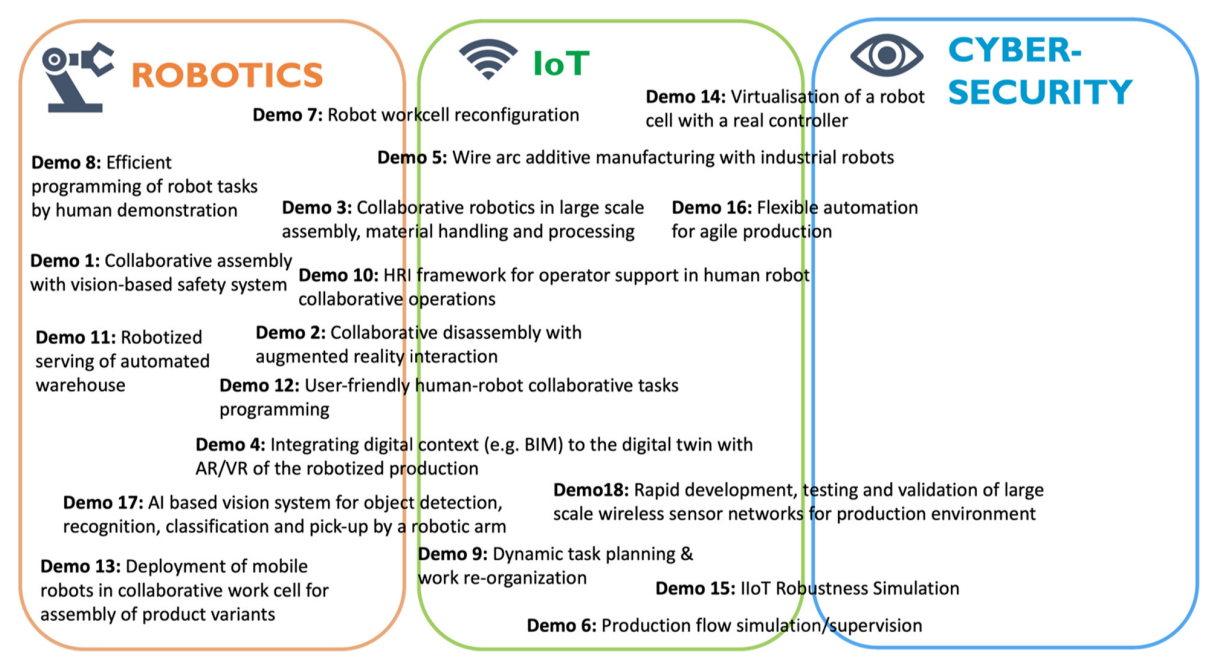

Fig. 2. The positioning of internal use-case demonstrations in the TRINITY project

The approach for building use-case demonstrations is divided into 4 stages.

- The first 12 months of the TRINITY was dedicated to the research and development of the internal use case demonstrations. This stage ensured that the project'a Internal demonstrations meet the market requirements and are appealing to the small batch and mainstream manufacturing and robotics sectors. 
- The internal use case demonstrations and their modules will be enhanced and modified to fit the companies interests during year 2. Extensive testing will be carried out in all aspects related to the performance, effectiveness, deployability, and safety. This year is dedicated also for running the first open call and development of business plan for DIH and individual exploitable results.

- In the third phase the new demonstrations from the open call will be included to the digital catalog the TRINITY develops for marketing and dissemination purposes. This is expected to increase the visibility of possible robotics-related solutions among SMEs. During this year the second open call will be started.

- The final phase includes the finalisation of education management system and second round of 3rd party use-case demonstrations will be in the main focus. The results from both of these will be assessed and included to the business planning of DIH, internal demonstration and 3rd party demonstrations.

\subsection{Concept for Approaching the Industrial Partners}

The project aims to attract the new industrial companies by offering funding opportunities via open calls. The main aim is bring together partners who have not collaborated previously but wish to try collaboration in a European wide scheme. The open call for demonstrations is done in collaboration with participating cluster organisations and with an external advisory board in order to ensure that the maximum number of companies around Europe are reached. The consortium members will set up several web lectures on how to apply for funding for the demonstrations. It is expected that in the first open call there will be 30-50 applications for funding, majority focusing on the adoption and/or development of robotics technologies.

\section{Conclusions}

The main aim of the paper was to discuss of the future needs of the European industry and of the possibilities for improved technology transfer methods deployed in previous years. According to the visions referred there is a strong belief that with robotics European industry can increase the product quality, production capacity and production predictability, and with by applying IOT and AI the horizontal and vertical transparency of supply network could be increased while non-value added time in order-delivery processes could be decreased. The third expectation for the emerging ecosystems is to support the technology transfer from science to industry, support the resource and capacity sharing among the companies, and support life-long learning of working personnel. The population of the Europe is not growing, and at the same time there is a lack of interested and qualified workers coming to the markets. Partly this is because of demographics, and partly due to the decreasing interest towards STEM topics among younger generation. The Digital Innovation Hubs -concept in general aims to support the knowledge, technology and competence transfer and sharing among European SMEs. 
This paper also provided a short overview of concrete and recent actions regarding the robotics ecosystem building and/or building of Digital Innovation Hubs having (or supporting projects with) a cascade funding dimension for experiments and/or demonstrators. The time perspective was set to projects funded from 2016 onwards. As the Fig. 1 shows there is a good number of projects running different types of cascade programs. The main idea in these is to bring H2020 framework programmes closer to the European SMEs and support them in digital transformation and adoption of emerging technologies. A more detailed overview of the project TRINITY DIH project was included into the paper. The TRINITY aims to increase the interest of the companies with different use-case demonstrations (or prototypes) that could be replicated to industrial setting. While there are no concrete KPIs of the success the previous cascade funding projects show increasing interest from the SMEs. Secondly the TRINITY DIH project intents to deliver both realistic use-cases and associated training material for using novel robotics technologies in the manufacturing.

Acknowledgements. This project has received funding from the European Union's Horizon 2020 research and innovation programme under grant agreement No 825196.

\section{References}

1. DIH agROBOfood - Business-Oriented Support to the European Robotics and Agri-food Sector, towards a network of Digital Innovation Hubs in Robotics, GA 825395. https://agrobofood.eu/

2. BDVA - euRobotics, Joint Vision Paper for an Artificial Intelligence Public Private Partnership (AI PPP), p. 7 (2019)

3. Chryssolouris, G., Mavrikios, D., Rentzos, L.: The teaching factory: a manufacturing education paradigm. In: 49th CIRP Conference on Manufacturing Systems, Procedia CIRP (2014)

4. European Commission, Statistics. https://cordis.europa.eu. Accessed 31 Jan 2020

5. COVR - Being safe around collaborative and versatile robots in shared spaces, GA 779966. https://safearoundrobots.com/. Accessed 30 Jan 2020

6. De Santis, A., Siciliano, B., De Luca, A., Bicchi, A.: An atlas of physical humanrobot interaction. Mech. Mach. Theory 43(3), 253-270 (2008)

7. DIHsquared. https://dih-squared.fundingbox.com/

8. DIHNET - Next Generation European DIH Network, GA 825640. http://www. dihnet.eu. Accessed 31 Jan 2020

9. European Commission, DG Connect, Digital Innovation Hubs, Draft (2018). https://ec.europa.eu/futurium/en/system/files/ged/digital_innovation_hubs_in_ digital_europe_programme_final2_december.pdf

10. European Commission, DG on Directorate-General for Education, Youth, Sport and Culture, 2019, Education and Training, MONITOR 2019, Country analysis. https://ec.europa.eu/education/sites/education/files/document-library-docs/ volume-2-2019-education-and-training-monitor-country-analysis.pdf

11. Eurostat Explained, Manufacturing statistics - NACE Rev. 2 (2019). https:// ec.europa.eu/eurostat/statistics-explained/index.php/Manufacturing_statistics_-_ NACE_Rev._2\#Sectoral_analysis 
12. Esmera - European SMEs Robotics Applications, GA 780265. http://www.esmeraproject.eu/welcome/. Accessed 31 Jan 2020

13. euRobotics, Robotics 2020 - Strategic Research Agenda for Robotics in Europe, p. $100(2013)$

14. European Commission, People in the EU - statistics on demographic changes. https://ec.europa.eu/eurostat/statistics-explained/index.php/People_in_ the_EU_-_statistics_on_demographic_changes

15. Halme, R.-J., Lanz, M., Kamarainen, J., Pieters, R., Latokartano, J., Hietanen, A.: Review of vision based safety systems for human-robot collaboration. In: CIRP CMS 2018, Procedia Manufacturing, Stockholm, May 2018

16. Hamalainen, R., Wever, B., Malin, A., Cincinnato, S.: Education and working life: VET adults' problem-solving skills in technology-rich environments. Comput. Educ. 88(October), 38-47 (2015)

17. DIH Hero - Digital Innovation Hubs in Healthcare Robotics, GA 825003. https:// dih-hero.eu. Accessed 31 Jan 2020

18. Hietanen, A., Pieters, R., Lanz, M., Latokartano, J., Kamarainen, J.-K.: ARbased interaction for human-robot collaborative manufacturing. J. Robot. Comput. Integr. Manuf. 63, 18 (2019)

19. Horse - Smart integrated Robotics system for SMEs controlled by Internet of Things based on dynamic manufacturing processes, GA 680734. http://www.horseproject.eu/. Accessed 31 Jan 2020

20. International Federarion of Robotics, Statistics, 2017 report (2017). https://ifr.org

21. International Labour Office, A Skilled Workforce for Strong, Sustainable and Balanced Growth: A G20 Training Strategy, ISBN 978-92-2-124278-9 (2010)

22. Jarvenpaa, E., Siltala, N., Lanz, M.: Implementation of capability matchmaking software facilitating faster production system design and reconfiguration planning. J. Manuf. Syst. 53, 261-270 (2019)

23. Lanz, M., Jarvenpaa, E.: Social sustainability and continuous learning in the circular economy framework. Leal Filho, W., Azul, A., Brandli, L., Ozuyar, P., Wall, T. (eds.) Responsible Consumption and Production, Encyclopedia of the UN Sustainable DevelopmentGoals. Springer, Cham (2019). https://doi.org/10.1007/9783-319-71062-4_35-1

24. L4MS - Logistics for Manufacturing SMEs, GA 767642. http://www.14ms.eu/. Accessed 26 Jan 2020

25. Manufuture, ManuFUTURE Vision 2030 - A Competitive, Sustainable and Resilient European Manufacturing, Report of the ManuFUTURE ? EU High-Level Group, p. 37, April 2018

26. Michalos, G., Makris, S., Tsarouchi, P., Guasch, T., Kontovrakis, D., Chryssolouris, G.: Design considerations for safe human-robot collaborative workplaces. Procedia CIRP 37, 248-253 (2015)

27. Michalos, G., Fysikopoulos, A., Makris, S., Mourtzis, D., Chryssolouris, G.: Multi criteria assembly line design and configuration - an automotive case study. CIRP J. Manuf. Sci. Technol. 9, 69-87 (2015)

28. Micro-ROS - Platform for seamless integration of resource constrained devices in the robot ecosystem, GA 780785. https://micro-ros.github.io. Accessed 31 Jan 2020

29. Reconcell - A Reconfigurable robot workCell for fast set-up of automated assembly processes in SMEs, GA 680431. http://www.reconcell.eu/index.html. Accessed 26 Jan 2020

30. DIH Rima - Robotics for Infrastructure Inspection and MAintenance, GA 824990. https://rimanetwork.eu/. Accessed 31 Jan 2020 
31. Robotics Tomorrow, Collaborative Robots Market - Shifting Technology from Robots to Cobots http://www.roboticstomorrow.com/news/2016/11/03/ collaborative-robots-market-shifting-technology-from-robots-to-cobots/9049/ (2016)

32. RobotUnion - Stimulate ScaleUps to develop novel and challenging TEchnology and systems applicable to new Markets for ROBOtic soLUTIONs, GA 779967. https://robotunion.eu. Accessed 31 Jan 2020

33. RODIN - RObotics Digital Innovation Network, GA 825263. https://rodinrobotics.eu/. Accessed 29 Jan 2020

34. EmerJ, Global Competition Rises for AI Industrial Robotics (2019). https://emerj. com/ai-sector-overviews/global-competition-rises-ai-industrial-robotics/

35. TRINITY - Digital Technologies, Advanced Robotics and increased Cyber-security for Agile Production in Future European Manufacturing Ecosystems, GA 825196. https://www.trinityrobotics.eu

36. Vogel, C., Walter, C., Elkmann, N.: Safeguarding and supporting future humanrobot cooperative manufacturing processes by a projection- and camera-based technology. Procedia Manuf. 11(June), 39-46 (2017)

Open Access This chapter is licensed under the terms of the Creative Commons Attribution 4.0 International License (http://creativecommons.org/licenses/by/4.0/), which permits use, sharing, adaptation, distribution and reproduction in any medium or format, as long as you give appropriate credit to the original author(s) and the source, provide a link to the Creative Commons license and indicate if changes were made.

The images or other third party material in this chapter are included in the chapter's Creative Commons license, unless indicated otherwise in a credit line to the material. If material is not included in the chapter's Creative Commons license and your intended use is not permitted by statutory regulation or exceeds the permitted use, you will need to obtain permission directly from the copyright holder. 